\title{
Charte «Collaboration entre les professionnels de la santé»
}

Académie Suisse des Sciences Médicales (ASSM)

Un commentaire de la FMH se trouve à la page 1806 .
Correspondance: Académie Suisse des Sciences Médicales (ASSM) Petersplatz 13 CH-4051 Bâle mail[at]samw.ch

\section{Préambule}

Depuis 1999, l'Académie Suisse des Sciences Médicales (ASSM) se préoccupe des buts et missions de la médecine dans le cadre de son projet «La médecine en Suisse demain». En 2007, un groupe de travail avait publié le rapport «Les futurs profils professionnels des médecins et des infirmiers dans la pratique ambulatoire et clinique». L'une des recommandations émises dans ce rapport concernait l'élaboration d'une «charte» pour la collaboration entre les professionnels de la santé. Des entretiens avec des experts ont confirmé la pertinence d'une telle charte, dans le sens d'un soutien face aux défis actuels et futurs du système de santé.

La présente charte devrait permettre a) d'optimiser le traitement des patients, b) de garantir la prise en charge compte tenu du manque croissant de professionnels de la santé et c) de soutenir le travail des professionnels de la santé. Pour ce faire, la charte se réfère à des initiatives similaires à l'étranger.

La charte de l'ASSM s'adresse en priorité aux professionnels, aux associations et aux institutions du système de santé; elle entend refléter l'esprit de coopération des professionnels de la santé et constitue la base du virage culturel souhaité. Elle entend également soutenir les nombreuses initiatives du système de santé qui exigent une coopération entre les professionnels de la santé (par exemple les stratégies en matière de soins palliatifs, de démence et contre le cancer).

Une collaboration fructueuse et réussie entre professionnels de la santé exige également des conditions-cadres adéquates; dès lors, la politique, l'administration et les assurances sont, elles aussi, invitées à se référer à la charte.

La charte est un document dynamique; elle évoluera dans le temps et peut être adaptée aux nouvelles données. Les destinataires sont invités à considérer ce processus d'évolution et de transformation comme une chance et à y participer activement.

\section{Principes}

Les représentants des professions de la santé s'engagent pour une prise en charge optimale et de haute qualité, adaptée aux besoins et aux attentes de la population.

Les patients se situent au cœur de la prise en charge. Cela signifie que les prestations éducatives, consultatives, préventives, diagnostiques, thérapeutiques, soignantes, réhabilitatives et palliatives de tous les professionnels de la santé impliqués doivent être coordonnées. La responsabilité est assumée selon les compétences professionnelles. L'information réciproque est garantie à tout moment; le travail est basé sur le respect mutuel et sur des standards reconnus et définis de concert.

L'objectif d'une telle prise en charge est d'assurer à toute la population un accès équitable à des prestations médicales de bonne qualité, satisfaisantes pour les patients et les collaborateurs et efficientes; elle s'inscrit ainsi dans le concept d'une «médecine durable» orientée vers des critères basés sur des preuves, l'éthique et l'économie.

\section{Eléments clés et obligations}

\section{La collaboration interprofessionnelle inclut} le patient comme partenaire

De plus en plus, les patientes et patients informés et leurs proches souhaitent exercer un rôle actif au sein du système de santé et participer aux décisions relatives à la thérapie et aux soins. En tant qu'«experts de soi-même», ils partagent dans une large mesure la responsabilité de la qualité des traitements et du résultat.

\section{Les professions de la santé clarifient et défi-} nissent leurs compétences et leurs responsabilités, pour les situations concrètes, dans un processus de concertation commun; en cas de chevauchement des compétences, les responsabilités doivent être explicitement définies.

Une partie de ce processus de clarification peut être définie dans des directives et des définitions générales, acceptées à l'unanimité; pour être pertinente, l'autre partie est définie au sein de chaque institution ou organisation (hôpital, service ambulatoire / policlinique, centre de santé, cabinet de groupe, réseau, soins à domicile, soins stationnaires à long terme, entre autres). La prise de responsabilités supplémentaires requiert un cadre clairement défini (par exemple en cas de compétence élargie pour le diagnostic, la prescription et la facturation pour les professions de la santé concernées).

\section{Les différentes tâches dans les processus de} prise en charge sont exécutées selon les besoins des patients et de leurs proches et selon les compétences professionnelles nécessaires.

La répartition des tâches ne répond pas à des critères hiérarchiques et/ou à des profils professionnels tradi- 
tionnels. Selon la situation, les groupes professionnels sont prêts à déléguer des tâches ou à endosser de nouvelles responsabilités. Cela signifie que les professionnels de la santé interviennent en fonction de leurs compétences et de leurs capacités spécifiques et assument la responsabilité de leurs actes.

4. Les associations professionnelles élaborent des directives interprofessionnelles communes en tenant compte des recommandations internationales; au niveau institutionnel, les différents groupes professionnels complètent ces directives par des standards interprofessionnels communs. Ces directives et standards institutionnels servent de garde-fous; pour gagner la confiance mutuelle, il est important de les respecter. Un écart par rapport aux directives et standards est possible, mais doit être justifié.

\section{Des modules communs sont élaborés, tant pour la formation prégraduée que pour la} formation postgraduée. Les institutions de formation prégraduée et postgraduée des différentes professions de la santé s'organisent en réseau.

Dans l'optique d'une pratique interprofessionnelle, les principaux éléments interprofessionnels et coopératifs sont intégrés dans les formations pré- et postgraduées des professions de la santé. En plus des enseignements spécifiques, des éléments de base communs (par exemple des connaissances concernant la santé et la maladie, ainsi que les principes de la coopération interprofessionnelle en plus de la communication, de l'éthique, de l'économie et de la politique de la santé) sont transmis aux étudiants dans le cadre de modules communs.
6. L’apprentissage à vie et la justification des compétences et aptitudes constituent une évidence pour toutes les associations professionnelles concernées et sont encouragés par les employeurs et les associations professionnelles.

En médecine, le savoir et l'éventail des activités en début de carrière diffèrent de ceux en fin de carrière. Une formation ciblée, tout au long de la vie professionnelle, l'acquisition de nouvelles compétences et le souci constant de garantir des prestations de qualité dans son propre domaine d'activité: telles sont les conditions requises pour l'exercice des professions de la santé. Des offres adéquates de formation continue sont accessibles à tous les groupes professionnels concernés et - lorsque cela s'avère pertinent - sont organisées par des représentants des différents groupes professionnels.

\section{Les modèles de travail sont conçus de telle} façon qu'ils encouragent et soutiennent une prise en charge intégrée.

Les organisations de la santé qui fonctionnent comme centres regroupent et coordonnent tous les groupes professionnels impliqués dans un processus de prise en charge et offrent ainsi de bonnes conditions pour une collaboration compétente; elles servent également de plaque tournante pour la prise en charge médicale régionale. Il est possible de faire appel à d'autres partenaires de la région par l'intermédiaire d'un réseau. Afin de garantir un échange d'informations continu, il est indispensable d'avoir recours aux technologies de l'information et de la communication (eHealth). Des infrastructures adaptées favorisent les rencontres informelles entre les différents groupes professionnels et une communication transparente.

Indications concernant l'élaboration de cette charte

Le groupe de travail qui a élaboré cette charte est constitué des personnalités suivantes:

Dr Werner Bauer, Président SIWF, Küsnacht ZH (Président); Dr Hermann Amstad, Secrétaire général de l’ASSM, Bâle; Marie-Anne Becker, Hôpital Neuchâtelois, Neuchâtel; Dr Manuela Eicher, Haute Ecole de Santé HES-SO, Fribourg; Prof. Viola Heinzelmann, Universitäts-Frauenspital, Bâle; Dr Isabelle Meier, mediX Gruppenpraxis, Zurich; Maja Mylaeus-Renggli, Spitex Verband Schweiz, Berne; Dr Hans Neuenschwander, IOSI/EOC, Bellinzona; Prof. Cornelia Oertle, Fachhochschule Gesundheit, Berne; Dr Pierre-Yves Rodondi, Spécialiste en Médecine interne générale, Pully; Martine Ruggli, pharmaSuisse, Liebefeld; Prof. Astrid Schämann, ZHAW, Winterthour; Dr Beat Sottas, Careum, Zurich; Prof. Peter M. Suter, vice-président ASSM Presinge.

Le groupe de travail s'est réuni à plusieurs reprises, à partir du mois d'avril 2013, pour réfléchir à cette thématique. Après une étude de la littérature et des discussions approfondies, il a rédigé un premier projet de cette charte; début octobre, ce projet a été commenté dans le cadre d'auditions d'experts choisis (Dr François Héritier, Président SSMG, Courfaivre; Roland Paillex, Président physioswiss, Lausanne; Pierre Théraulax, Président ASI-SBK, Berne; lic. oec. Rita Ziegler, Directrice de l'hôpital universitaire Zurich; Dr Alan Niederer, journaliste scientifique, Zurich; lic. rer. pol. Michael Jordi, Secrétaire central CDS, Berne). Une version remaniée a ensuite été soumise à une large consultation publique. Si la majorité des prises de position ont salué l'élaboration d'une charte, un certain scepticisme était néanmoins perceptible au sein du corps médical et, par ailleurs, de nombreuses institutions auraient souhaité être davantage impliquées dans l'élaboration de cette charte. Egalement les propositions de mise en œuvre ont fait l'objet de critiques. Le groupe de travail a alors organisé un colloque à Berne, en août 2014, pour recueillir les attentes des différents protagonistes. Suite à cet échange, une version finale a été rédigée par le Comité de direction de I'ASSM puis discutée lors de sa séance du 3 novembre 2014 et définitivement approuvée. 


\section{Les structures dirigeantes sont organisées selon les objectifs.}

Les structures dirigeantes des institutions de la santé sont définies en fonction d'aspects objectifs sans tenir compte des questions de statut; elles peuvent ainsi soutenir efficacement le travail interprofessionnel. Les personnes qui assument des fonctions dirigeantes au sein d'une équipe interdisciplinaire doivent justifier de la compétence correspondante.

\section{L'assurance qualité et l'évaluation de l'utilité pour le patient et la société font partie des valeurs professionnelles de tous les groupes professionnels.}

L'institution et la mise en œuvre de la collaboration entre toutes les professions médicales doivent être accompagnées d'une recherche adéquate sur les services de santé.

\section{Mise en œuvre}

- Les associations professionnelles, les institutions de la santé (par exemple hôpitaux, réseaux, soins à domicile, soins stationnaires de longue durée) et les instituts de formation du domaine de la santé engagent avec leurs organes une discussion approfondie concernant la présente charte et sa signification pour leur propre organisation.

- Les associations professionnelles lancent des projets pilotes pour la définition des compétences et des responsabilités dans des situations concrètes, l'élaboration de directives interprofessionnelles et l'organisation de sessions de formations communes.

- Les associations professionnelles s'engagent de concert pour les adaptations des lois, des ordonnances et des tarifs, considérées comme nécessaires.

- La charte sert d'outil d'enseignement et de base de discussion dans les formations prégraduée, postgraduée et continue, ainsi que d'incitation pour le développement commun de programmes d'études.

- La charte constitue la base du contact institutionnalisé des associations et instituts de formation.

- L'ASSM assure le suivi de l'évolution de la charte: elle répertorie l'ensemble des initiatives et projets - dans le sens d'un monitoring - et organise au plus tard deux ans après la publication de la charte une rencontre entre les différents protagonistes dans le but a) d'échanger les expériences, b), le cas échéant, d'adapter la charte et c) de décerner un prix aux projets de coopération particulièrement réussis.

\section{Références}

- American College of Physicians (ACP). The Advanced Medical Home: A Patient-Centered, Physician-Guided Model of Health Care; 2006.

- World Health Professions Alliance (WHPA). Joint Health Professions Statement on Task Shifting; 2008

- World Health Organisation (WHO). Task shifting: rational redistribution of tasks among health workforce teams; 2008

- American College of Physicians (ACP). Nurse Practitioners in Primary Care; 2009.

- Frenk J et al. Health Professionals for a new century: transforming education to strengthen health systems in an interdependent world. Lancet. Report; 2010.

- WHO. Framework for action on interprofessional education and collaborative practice. Geneva; 2010.

- Robert-Bosch-Stiftung: Memorandum «Kooperation der Gesundheitsberufe»; 2011.

- Schweizerische Akademie der Medizinischen Wissenschaften (SAMW). Die zukünftigen Berufsbilder von Ärztinnen und Pflegenden - Bericht und Kommentar; 2011.

- Arbeitsgruppe BAG/GDK: Neue Versorgungsmodelle für die medizinische Grundversorgung; 2012.

- Witte F. Mehr kooperieren statt delegieren. Schweiz. Ärzteztg. 2012; 93(22):809-11.

- American Medical Association. The structure and functioning of interprofessional health care teams. Joint report of the Council of Medical Education and the Council Medical Service. Chicago; 2012.

- Ono T, Lafortune G, Schoenstein M. Health Workforce Planning in OECD Countries: a Review of 26 Projection Models from 18 Countries. OECD Health Working Paper No. 62; 2013.

- Plattform «Zukunft Ärztliche Bildung». Bericht «Interprofessionalität»; 2013.

- Donelan K et al. Perspectives of Physicians and Nurse Pratitioners on Primary Care Practice. NEJM. 2013;368:1898-1906.

- Iglehart J. Expanding the Role of Advanced Nurse Practitioners - Risk and Rewards. NEJM. 2013;368:935-41.

- APPG. All the Talents. How new roles and better teamwork can releasepotential and improve health services. London: All-Party Parliamentary Group; 2013.

- Careum. Neuausrichtung der Ausbildung für Gesundheitsberufe. Zürich; 2013.

- Sottas B et al. Umrisse einer neuen Gesundheitsbildungspolitik. Zürich: Careum Verlag; 2013.

- European Interprofessional Practice and Education Network (EIPEN): Charter für Interprofessional Practice and Education; 2014.

- Robert Bosch Stiftung. Gesundheitsberufe neu denken. Stuttgart; 2014

- Walkenhorst U, Mahler C, et al. Interprofessionelle Ausbildung in den Gesundheitsberufen. Positionspapier der Gesellschaft für medizinische Ausbildung (GMA). Erlangen; 2014. 